\title{
TRAUMATISMO CARDÍACO PENETRANTE EN PEDIATRÍA: PRESENTACIÓN DE CASO, HOSPITAL INFANTIL DR. ROBERT REID CABRAL (HIRRC), REPÚBLICA DOMINICANA
}

\section{Penetrating Cardiac Trauma in Children: Case Presentation, Hospital Infantil Dr. Robert Reid Cabral, Dominican Republic.}

\author{
Cristina Contreras ${ }^{1 *}$, Lisandra Corona ${ }^{2 * *}$, Norys Domínguez ${ }^{3 * * *}$, \\ Carolina Almonte ${ }^{3 * * *}$, Ernesto Taveras ${ }^{3 \Delta}$, Ricardo Rojas $^{4 \forall}$, Miguel Mejía-Sang $^{5 \Omega}$
}

Recibido: diciembre 15, 2018 - Aprobado: enero 24, 2019

Cómo citar: Contreras C, Corona L, Domínguez N, Almonte C, Taveras E, Rojas R, Mejía Sang M. Traumatismo cardíaco penetrante en pediatría: presentación de caso, Hospital Infantil Dr. Robert Reid Cabral (HIRRC), República Dominicana. cysa [Internet]. 26 de julio de 2019 [citado 26 de julio de 2019];3(2): 77-83. Disponible en: https://revistas.intec.edu.do/index.php/cisa/article/ view/1482

\section{Resumen}

Introducción: el traumatismo cardíaco es una condición poco frecuente en edad pediátrica, ocurriendo en menos del $5 \%$ de traumatismos torácicos. Afecta más frecuentemente los ventrículos que las aurículas. Según el mecanismo de injuria puede ser: cerrado (como las contusiones torácicas) o penetrante (como las heridas por arma blanca o armas de fuego).

El siguiente documento tiene la intención de describir la presentación clínica y el manejo de un paciente pediátrico con traumatismo cardíaco penetrante; se pretendió realizar una revisión de literatura sobre otros casos de traumatismo cardíaco penetrante.

Presentación del caso: se expone el caso clínico de un paciente masculino de 14 años de edad, quien es referido al centro por historia de herida de arma blanca en región precordial izquierda. Se recibe con inestabilidad hemodinámica y hemitórax izquierdo hipodinámico; luego de realizar estudios de imagen y laboratorio se decide realizar toracocentesis. Ante evidente deterioro clínico, se decide realizar exploración quirúrgica, donde se halla laceración del

\begin{abstract}
Introduction: Cardiac trauma is a rare condition in pediatrics, occurring in less than $5 \%$ of thoracic trauma. It affects the ventricles more often than the atria. Depending on the mechanism of injury, it may be classified as closed (such as blunt chest traumas) or penetrating (such as wounds with a knife or guns).

The following document has the intention to describe the clinical presentation and management of a pediatric patient with a penetrating cardiac trauma, and it was intended to conduct a literature review on other cases of penetrating cardiac trauma.
\end{abstract}

Case presentation: We present the clinical case of a 14-year-old male, who is referred to the center with history of a knife wound in the left precordial region. He is received with hemodynamic instability and hypodynamic left hemithorax, and, after performing imaging and laboratory studies, it was decided to perform thoracentesis. In the presence of evident clinical deterioration, it was decided to perform surgical exploration, where laceration of the left ventricle and pericardial hematoma is evidenced, for
1. Médico Ayudante de Urgencias Pediátricas del Hospital Infantil Dr. Robert Reid Cabral (HIRRC), República Dominicana

2. Jefa de Emergencias Pediátricas del HIRRC

3. Residente de Emergenciología y Cuidado Crítico Pediátrico del HIRRC

4. Residente de Cirugía Pediátrica del HIRRC

5. Médico General

\footnotetext{
Correo-e de los autores:

*crismi117@hotmail.com

**1coronaq@gmail.com

*** anyolinadominguez@hotmail.com

****dralmontemejia@gmail.com

$\Delta$ ernesto_taveras28@hotmail.com $\forall$ rrojas_v@hotmail.com

$\Omega$ miguel.mejia.sang@gmail.com
} 


\section{Cristina Contreras, Lisandra Corona, Norys Domínguez, Carolina Almonte, Ernesto Taveras, Ricardo Rojas y Miguel Mejía-Sang}

ventrículo izquierdo y hematoma pericárdico, y se procede a drenar hematoma y cardiorrafia de ventrículo izquierdo.

Conclusión: luego de describir la presentación y manejo de nuestro paciente, llegamos a la conclusión de que la sospecha clínica y el diagnóstico y manejo precoz son imprescindibles para mejorar el pronóstico de estos traumatismos. Asimismo, entendemos pertinente la exploración quirúrgica temprana ante los casos de inestabilidad hemodinámica, evitando el retraso de un tratamiento oportuno.

Palabras clave: traumatismo cardíaco; inestabilidad hemodinámica; exploración quirúrgica; hematoma pericárdico; cardiorrafia.

\section{Introducción}

El traumatismo cardíaco es una condición poco frecuente en edad pediátrica, ocurriendo en menos del $5 \%$ de traumatismos torácicos ${ }^{1}$. Afecta más frecuentemente los ventrículos (35\% el ventrículo derecho y $25 \%$ el izquierdo) que las aurículas $(30 \%)$ y menos frecuentemente produce lesiones coronarias, valvulares o septales ${ }^{1,2}$.

El traumatismo del corazón integra básicamente dos grandes grupos según el mecanismo de injuria: 1) traumatismo cardíaco cerrado (como las contusiones torácicas) y 2) traumatismo cardíaco penetrante (como las heridas por arma blanca o armas de fuego) (Cuadro 1) $)^{1,3}$.

Cuadro 1. Clasificación de traumatismos cardíacos en base al mecanismo de injuria ${ }^{3}$

\begin{tabular}{|c|l|}
\hline \multicolumn{2}{|c|}{ Clasificación de traumatismos cardíacos } \\
\hline Penetrantes & Heridas por arma blanca, por armas de fuego. \\
\hline $\begin{array}{c}\text { Contusiones } \\
\text { (no } \\
\text { penetrantes) }\end{array}$ & $\begin{array}{l}\text { Accidente automovilístico, caídas desde } \\
\text { altura, accidente industrial (compresión), } \\
\text { explosión (explosivos, granadas), asalto (agra- } \\
\text { vado), fractura esternal o costal, recreacional } \\
\text { (eventos deportivos, corrida de toros). }\end{array}$ \\
\hline Iatrogénicos & Inducidos por catéteres o pericardiocentesis. \\
\hline Metabólicos & $\begin{array}{l}\text { Respuesta traumática a la lesión, síndrome de } \\
\text { respuesta inflamatoria sistémica. }\end{array}$ \\
\hline Otros & $\begin{array}{l}\text { Quemaduras, electricidad, facticio (aguja, } \\
\text { cuerpos extraños), embólicos. }\end{array}$ \\
\hline
\end{tabular}

which drainage of hematoma and left ventricle cardiorrhaphy were performed.

Conclusion: After describing the presentation and management of our patient, we conclude that clinical suspicion and early diagnosis and management are essential to improve the prognosis of these injuries. Likewise, we recognize the pertinence of early surgical exploration in cases of hemodynamic instability, avoiding the delay of a timely treatment.

Keywords: Cardiac trauma; hemodynamic instability; surgical exploration; pericardial hematoma; cardiorrhaphy.

Tomando en cuenta el grupo etario al que pertenecen los pacientes con traumatismo cardíaco, el mecanismo de la lesión puede variar: 0-4 años son frecuentes los accidentes en vehículo de automotor y la violencia infantil; a los 5-9 años son asociadas a atropellamiento; y entre 10-17 años son secundarias a la práctica de algunos deportes (patinaje, ciclismo), accidentes automovilísticos, violencia y suicidio ${ }^{1}$.

El traumatismo cardíaco demuestra una variable presentación, por lo que la sospecha diagnóstica es muy importante ante el primer contacto con el paciente ${ }^{1,4}$. En términos clínicos, las lesiones superficiales que sugieren el trayecto del proyectil o del arma blanca en el tórax y la inestabilidad hemodinámica nos dirigen al diagnóstico de esta emergencia ${ }^{1,3}$.

No obstante, la clínica de los pacientes pediátricos es diferente a los pacientes adultos, por lo que puede dificultarse el diagnóstico en este grupo etario ${ }^{2}$.

El curso clínico podría incluir desde pacientes asintomáticos (en aquellos cuyas heridas no son transmurales o son de reducido tamaño en el ventrículo izquierdo, que por su masa muscular pueden ser ocluidos o producir pequeños derrames pericárdicos que no resultan en cambios hemodinámicos o expresiones tardías), hasta alteraciones estructurales (como insuficiencia cardíaca aguda), alteraciones eléctricas 
(como arritmias y aparición de soplos) e inestabilidad hemodinámica (como choque hipovolémico) ${ }^{1,3,5}$.

El desenlace del manejo de los traumatismos cardíacos depende en mayor medida del tiempo transcurrido desde el momento de la agresión hasta la llegada a centros capacitados para el manejo quirúrgico de la condición y subsiguientes medidas de soporte, que de la disponibilidad de equipos de cirugía torácica o cardíaca ${ }^{3,6}$. Otro de los factores que inciden en gran medida en el resultado de esta emergencia es el lugar anatómico de la lesión, considerándose las heridas a la zona II de Murdock (área precordial, epigastrio y mediastino superior) como de alto riesgo (Cuadro 2) ${ }^{7,8}$.

Cuadro 2. Severidad del traumatismo cardíaco ${ }^{8}$

\begin{tabular}{|c|l|}
\hline \multicolumn{2}{|c|}{ Severidad del traumatismo cardíaco } \\
\hline Grado & \multicolumn{1}{c|}{ Descripción de la lesión } \\
\hline I & $\begin{array}{l}\text { Lesión cardíaca cerrada con cambios mínimos en el ECG. } \\
\text { Lesión pericárdica sin afectación cardíaca, taponamiento cardíaco o hernia cardíaca. }\end{array}$ \\
\hline II & $\begin{array}{l}\text { Lesión cardíaca cerrada con aparición de bloqueo cardíaco o cambios isquémicos sin fallo cardíaco. } \\
\text { Lesión miocárdica tangencial sin extensión hasta el endocardio o taponamiento. }\end{array}$ \\
\hline III & $\begin{array}{l}\text { Lesión cardíaca cerrada con extrasistolia ventricular multifocal o sostenida. } \\
\text { Lesión cardíaca con rotura del septo, insuficiencias de las válvulas pulmonar o tricúspide, disfunción del } \\
\text { músculo papilar u oclusión arterial coronaria sin signos de fallo cardíaco. } \\
\text { Lesión con desgarro pericárdico y hernia cardíaca. } \\
\text { Lesión cardíaca cerrada con fallo cardíaco. } \\
\text { Lesión abierta tangencial del miocardio sin afectación endocárdica, pero con taponamiento. }\end{array}$ \\
\hline IV & $\begin{array}{l}\text { Lesión cardíaca con rotura del septo, insuficiencias de las válvulas pulmonar o tricúspide, disfunción del } \\
\text { músculo papilar u oclusión arterial coronaria con signos de fallo cardíaco. } \\
\text { Lesión cardíaca con insuficiencia valvular mitral o aórtica. } \\
\text { Lesión cardíaca con afectación del ventrículo derecho o de una de las dos aurículas. }\end{array}$ \\
\hline VI & $\begin{array}{l}\text { Lesión cardíaca con oclusión de una arteria coronaria proximal. } \\
\text { Lesión cardíaca con perforación ventricular izquierda. } \\
\text { Lesión por estallido cardíaco con pérdida inferior al } 50 \text { \% del ventrículo derecho, aurícula derecha o } \\
\text { aurícula izquierda. }\end{array}$ \\
\hline V & $\begin{array}{l}\text { Lesión cerrada con avulsión cardíaca o herida penetrante con pérdida superior al } 50 \% \text { de una cámara. } \\
\text { Avance de un grado ante la presencia de heridas penetrantes múltiples sobre una o varias cavidades. }\end{array}$ \\
\hline & \begin{tabular}{c} 
II \\
\hline
\end{tabular}
\end{tabular}

Las complicaciones del traumatismo cardíaco dependen del mecanismo de la lesión, y pueden ser: taponamiento cardíaco (si existe una restricción al llenado de las cavidades cardíacas, y se presenta sin la clásica triada de Beck en niños), contusión cardíaca, lesión aórtica, daño cardíaco estructural (rotura cardíaca, fístulas de arterias coronarias, defectos septales y lesiones valvulares) ${ }^{1}$. Consecuentemente, los casos de trauma cardíaco penetrante presentan una alta mortalidad, que alcanza 70-80 \% de los casos ${ }^{2}$. El diagnóstico de un trauma cardíaco penetrante inicia con la sospecha clínica, pero aún no existen criterios diagnósticos claros para este tipo de emergencias $^{1,2,9}$. En pacientes hemodinámicamente estables, el ecocardiograma es el estudio de elección 


\section{Cristina Contreras, Lisandra Corona, Norys Domínguez, Carolina Almonte, Ernesto Taveras, Ricardo Rojas y \\ Miguel Mejía-Sang}

para detectar la severidad de la herida cardíaca, con una sensibilidad de $90 \%$ y especificidad de $97 \%$.

Dependiendo de la severidad del cuadro y la estabilidad hemodinámica, se utilizan subsiguientemente las enzimas cardíacas, radiografía de tórax, electrocardiograma (EKG), tomografía o angiografía y podría realizarse una ventana pericárdica si es necesario ${ }^{1,2}$.

El manejo de los traumatismos penetrantes del corazón es multidisciplinario, pero el tratamiento definitivo lo constituye la cardiorrafia a través de toracotomía o esternotomía ${ }^{10,11}$. La reanimación, la intervención quirúrgica inmediata, un apoyo intensivo perioperatorio enérgico, son factores fundamentales en el manejo de los casos de herida penetrante cardíaca y pueden disminuir significativamente la mortalidad de estos pacientes a un $3.4 \% \%^{3,9,11,12}$.

\section{Reporte del caso}

Paciente masculino de 14 años de edad, sin antecedentes mórbidos conocidos, quien es referido al centro con historia de haber presentado herida de arma blanca en región precordial izquierda secundaria en una disputa por un supuesto robo y llevado a un centro de su comunidad, donde se le realizan primeros auxilios y, debido a la inexistencia de Departamento de Cirugía Pediátrica, es referido a nuestro centro. Es recibido con los signos vitales de $37^{\circ} \mathrm{C}$ de temperatura corporal, $118 \mathrm{~L} / \mathrm{min}$ de frecuencia cardíaca, y 20 respiraciones/min de frecuencia respiratoria.

El examen físico mostraba un paciente alerta, consciente y orientado en persona, tiempo y espacio, 15/15 en escala de Glasgow. Presentaba hemitórax izquierdo hipoventilado y con pérdida de la continuidad de la piel en 5to espacio intercostal línea media clavicular (5EII LMC), murmullo vesicular disminuido en zona afectada y crepitantes en campo pulmonar izquierdo, taquipnea, taquicardia, saturación de oxígeno en $95 \%$, hipotensión arterial, palidez general, pulsos centrales y periféricos débiles, llenado capilar $>3$ segundos, y extremidades frías.

En la sala de emergencias se le coloca oxígeno por cánula nasal, se toman muestras sanguíneas para realización de analíticas y se medica con analgesia (ketorolaco), antibioterapia (ceftriaxona y amikacina) y toxoide tetánico y gammaglobulina antitetánica humana. Se administran 2 cargas de solución Lactato Ringer a $20 \mathrm{~mL} / \mathrm{kg}$, se transfunde con 2 unidades de paquetes globulares compatibles y se coloca sonda vesical sin evidencia de orina.

Los exámenes de laboratorio mostraron leucocitosis $(21 \times 10 / \mathrm{mm})$, granulocitos en $67.7 \%$, hemoglobina de $7.9 \mathrm{~g} / \mathrm{dL}$, y hematocrito de $24.2 \%$. En la radiografía de tórax postero-anterior se observa una radioopacidad en hemitórax izquierdo con borramiento de los ángulos costofrénico y costodiafragmáticos y un signo de la silueta, sugiriendo líquido en hemitórax izquierdo. (Figura 1).

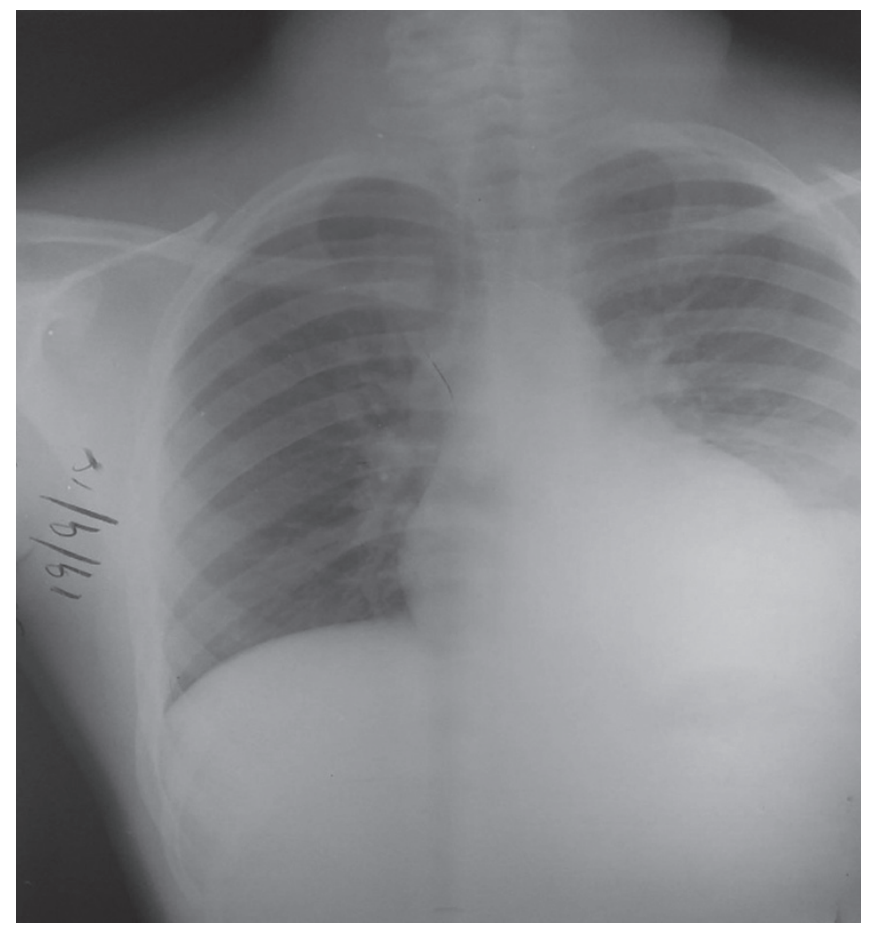

Figura 1. Radiografía de tórax antero-posterior.

Fuente: expedientes clínicos del Departamento de Cirugía Pediátrica del HIRRC. 


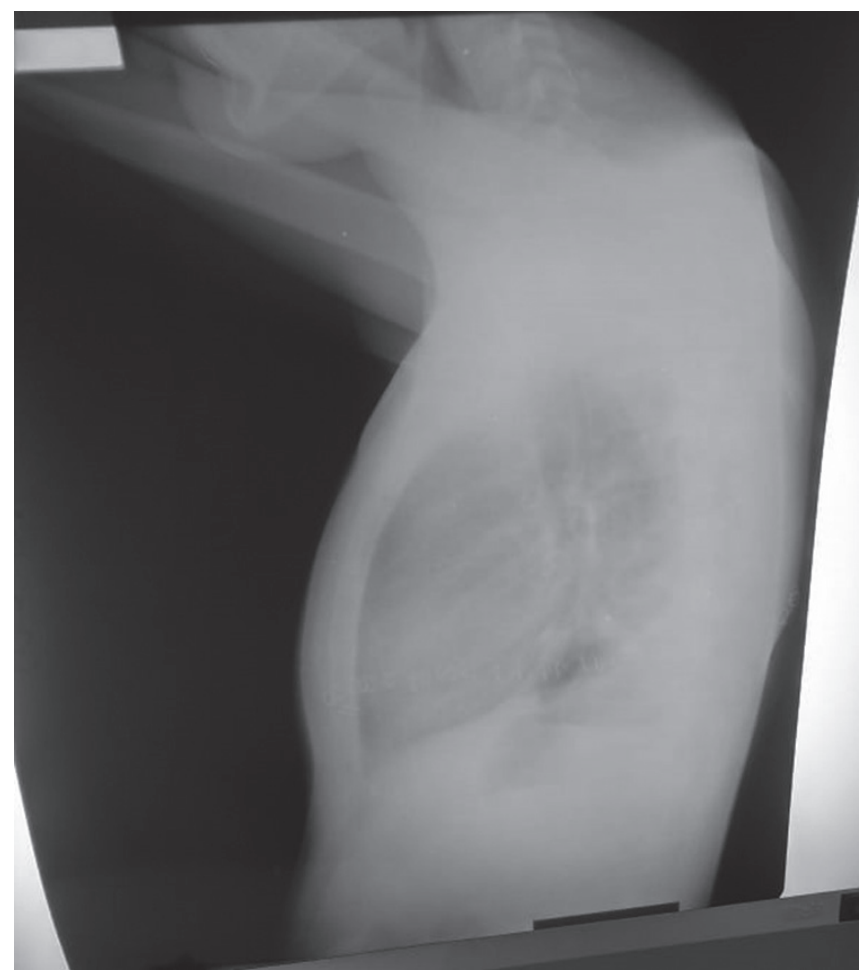

Figura 2. Radiografía de tórax lateral.

Fuente: expedientes clínicos del Departamento de Cirugía Pediátrica del HIRRC.

Se realiza toracocentesis y se obtiene contenido hemático. Ante la posibilidad de un hemotórax, se procede a colocar una sonda de pleurostomía izquierda, drenándose $350 \mathrm{~mL}$ de material hemático y se coloca bajo sello de agua.

Pese a los cuidados proporcionados, el paciente persiste con datos de choque hipovolémico que no responde a cargas ni hemoderivados, por lo que se procede a colocar un catéter venoso central femoral derecho y a administrar aminas vasoactivas (dobutamina y noradrenalina) y sedación (fentanilo y midazolam). Luego se introduce sonda nasogástrica y tubo endotraqueal, se inicia ventilación mecánica.

Consecuentemente, el Departamento de Cirugía Pediátrica decide el traslado del paciente a sala de operaciones para realizar exploración quirúrgica. Se realiza toracotomía anteroposterior izquierda donde se evidencia laceración del miocardio del ventrículo izquierdo, no transmural, y un hematoma pericárdico, por lo que se procede a realizar un drenaje de hematoma, corrección de laceración mediante cardiorrafia del ventrículo izquierdo, colocación de sonda de pleurostomía izquierda y realización de ventana pericárdica.

El paciente fue trasladado a unidad de cuidados intensivos, donde fue admitido en condiciones de sumo cuidado. Fue manejado con líquidos intravenosos, antibioterapia (vancomicina, meropenem), sulfato de magnesio, gluconato de calcio, ketorolaco y nalbufina, y ventilación mecánica. Luego de mostrar mejoría clínica y radiografías de tórax, ecocardiograma, hemograma y químicas dentro de los parámetros normales, se decide su egreso a los 6 días postquirúrgicos en condiciones generales estables.

\section{Discusión}

Los traumas cardíacos representan un porcentaje reducido del total de emergencias producidas por arma blanca, pero son las que se acompañan de una mayor mortalidad ${ }^{6}$. Las lesiones cardíacas penetrantes son una de las causas principales de muerte por violencia urbana ${ }^{13}$. Un manejo preoperatorio oportuno y eficaz, una intervención quirúrgica inmediata, una técnica quirúrgica de alta calidad y la capacidad de proveer un excelente cuidado crítico postoperatorio son cruciales para salvar la vida de un paciente con trauma cardíaco penetrante ${ }^{14}$.

En la población pediátrica, el traumatismo cardíaco es una entidad aún menos frecuente y representa un gran reto para pediatras y cirujanos pediátricos, pues la exploración y determinación de la severidad del caso son difíciles de realizar ${ }^{1,2}$.

A pesar de la limitada bibliografía de los casos reportados de traumas cardíacos penetrantes en pacientes pediátricos, es pertinente realizar una comparación con distintos casos de traumatismo cardíaco en adultos, para identificar posibles pautas para el mejoramiento del manejo de esta entidad. 


\section{Cristina Contreras, Lisandra Corona, Norys Domínguez, Carolina Almonte, Ernesto Taveras, Ricardo Rojas y Miguel Mejía-Sang}

Mahmudloo et al., describieron el caso de un paciente masculino de 12 años, el cual fue ingresado en un hospital de la ciudad de Urmia, Irán, por historia de haber recibido un trauma penetrante en esternón por una clavija de metal ${ }^{14}$. Al momento del ingreso, el único síntoma que presentó fue la incapacidad de tener una inspiración profunda y considerable dolor de pecho. Sus signos vitales se mantuvieron dentro de los parámetros normales, no presentó ruidos cardíacos o pulmonares anormales, y su EKG fue normal y ecocardiograma no detectó derrame pericárdico. Durante el transquirúrgico, se encontró el lugar de penetración en el tercio distal del esternón, además del lugar de penetración del ventrículo derecho, el cual fue expuesto y corregido. El curso postoperatorio transcurrió sin eventualidades, y al quinto día, luego de que los estudios ecocardiográficos no mostraran alguna anormalidad, el paciente fue dado de alta ${ }^{14}$.

En el estudio de casos realizado por Calderón et al., 7 de 8 pacientes diagnosticados con trauma penetrante cardíaco tuvieron como etiología el trauma por herida causada por arma blanca, cuatro pacientes se presentaron con signos de choque hipovolémico y en siete pacientes fue hallado hemotórax. Concluyeron que el ecocardiograma pierde exactitud en casos de hemotórax y ventilación mecánica, por lo que es pertinente el abordaje transesofágico, si la estabilidad hemodinámica lo permite ${ }^{12}$.

Van Heerden et al. reportaron el caso de una paciente femenina de dos ańos de edad, la cual se presentó a sala de emergencias una hora después de haber recibido una puñalada en el precordio ${ }^{15}$. A la inspección se observaron dos heridas penetrantes precordiales en el hemitórax izquierdo, sin presentar otras lesiones. Aunque estuvo hemodinámicamente estable al momento del ingreso, manifestó signos de deterioro después de media hora. La radiografía de tórax no mostró hemotórax o neumotórax. Se realiza ecografía que inicialmente no muestra derrame pericárdico, pero luego se evidenció un aumento progresivo del mismo. En la intervención quirúrgica se observa laceración de $0.5 \mathrm{~cm}$ en el ventrículo izquierdo, la cual fue corregida. Una semana después la paciente fue dada de alta, sin eventualidades ${ }^{15}$.

En cuanto al caso reportado, el paciente se mostró hemodinámicamente inestable a su llegada, con evidente shock hipovolémico, y con datos clínicos y radiográficos de hemotórax, por lo que se procede a administrar cargas, hemoderivados, aminas vasoactivas, antibioterapia, analgesia y sedación. Conjuntamente se le colocó sonda vesical y nasográstrica, tubo endotraqueal y ventilación mecánica y, además, una sonda de pleurostomía.

No obstante, el paciente no estaba respondiendo adecuadamente a las medidas terapéuticas y continuaba presentando signos de inestabilidad, por lo que fue trasladado a la sala de operaciones para realizar una exploración quirúrgica y evaluar la condición del paciente. Se pudo evidenciar una laceración del miocardio del ventrículo izquierdo y pudo ser corregido inmediatamente, llevando al paciente a la mejoría de su cuadro clínico y su egreso a su sexto día postquirúrgico.

\section{Conclusión}

El trauma cardíaco penetrante es una entidad muy poco frecuente en edad pediátrica y está acompañada de una alta mortalidad si no es diagnosticada a tiempo y manejada correctamente. Es por esto que la agudeza clínica es primordial ante un caso de lesión penetrante en el área precordial.

Consideramos importante la necesidad de realizar protocolos diagnósticos y terapéuticos integrales, que inicien desde el momento del trauma en el ambiente extrahospitalario hasta el manejo efectivo de esta emergencia en hospital. Esto hace imprescindible la difusión dentro de la comunidad médica, desde los proveedores de transporte de pacientes hasta los emergenciólogos, pediatras y cirujanos, del manejo efectivo de esta emergencia, que permitiría una oportuna atención y disminución de la mortalidad en estos pacientes. 
Asimismo, entendemos pertinente la exploración quirúrgica temprana ante los casos de inestabilidad hemodinámica, asegurando una apropiada evaluación de la severidad de cada caso, y evitando el retraso de un tratamiento oportuno.

\section{Bibliografía}

1. Reynoso-Montesinos JG, Peña-Becerra OG, SalgadoArzate SJ, Tarelo-Saucedo, JM. Traumatismo cardíaco en pediatría. Rev Esp Méd Quir. 2014;19: 332-5.

2. Peralta Sánchez JA, Correa Mazuera C, Luengas Monroy JP. Trauma cardíaco penetrante por arma de fragmentación en una lactante. Univ. Méd. 2015;56(1): 104-9.

3. Bernal-Reyes MP, Hernández-Avedaño ML, Launizar-García ME, Cuenca-Dardón JF. Heridas penetrantes de corazón. Reporte de un caso y revisión de la literatura. Rev Mex Anest. 2008;31(2): 146-50.

4. Gómez G, Hola J. Trauma penetrante cardíaco en la unidad de emergencia del Hospital Carlos Van Buren. Rev Chil Cir. 2009;61(5): 453-7.

5. Pereira Warr S, Chavarriaga Zapata JC. Traumatismo cardíaco con lesión del sistema de conducción, supervivencia de un paciente. Cir Cardiov. 2017;24(6): 350-2.

6. Álvarez Fernández JA, Delgado Millán MA, Alted López EJ. Heridas cardíacas por arma blanca. Emergencias. 1988;(1): 31-5.

7. Liberto D, Buela E, Mauri V, Volonté P, Huaier F, Fiorentino J. Traumatismo cardíaco en pediatría. Rev Hosp Niños BAires. 2008;50(227): 64-9.

8. The American Association for the Surgery of Trauma. Heart Injury Scale. [Online] Available from: http://www.aast.org/library/traumatools/ injuryscoringscales.aspx\#heart [Accesed $28^{\text {th }}$ January 2019].
9. De Raet J, Mees U, Vandekerkhof J, Hendrikx M. Penetrating pediatric cardiac trauma caused by fall on a pencil with a normal echocardiography. Interact Cardiovasc Thorac Surg. 2004;3(4): 634-6.

10. Castañeda E, Bazan N, Vásquez J, Delgado H. Trauma cardíaco penetrante. Experiencia en el Hospital Nacional Cayetano Heredia 1984-1993. Rev Med Hered. 1994;5: 192-7.

11. Göz M, Cakir Ö, Eren MN. Penetrating cardiac trauma in children. Ulus Travma Acil Cerrahi Derg. 2010;16(3): 220-4.

12. Calderón Villa F, Romero Vaca, M, Zapata Ibarra M, Bernal Rodríguez C. Manejo del trauma cardíaco penetrante, experiencia Hospital Carlos Andrade Marín. Revista CIEZT Clínica y Cirugía. 2014;12(1): 43-8.

13. Castriconi M, Festa P, Bartone G, Maglio MD, Vicenzo L, Papaleo D, Severino BU, ClementeM, Martino A. Penetrating cardiac injuries. Two case reports. Ann Ital Chir. 2013 Apr 24;84(ePub). pii: S2239253X13020987. PubMed PMID: 23877433. Available from: https://www.ncbi. nlm.nih.gov/pubmed/23877433

14. Mahmudloo R, Khosroshahi AJ, Gheibi S. Successful removal of a Rivet from the Heart of a boy with Penetrating Cardiac Injury: A Case Report and Literature Review. Int J Pediatr. 2014;2(4.2): 1-4.

15. Van Heerden YL, Solomon Z, Loveland J. Stab wound to the heart in a two year old. J Pediatr Surg Case Rep. 2018;32: 21-2.

16. Tang AL, et al. Postdischarge complications after penetrating cardiac injury. A Survivable Injury with a High Postdischarge complication rate. Arch Surg. 2011;146(9): 1061-6. 